\title{
Potentially inappropriate medication in the elderly in Germany: an economic appraisal of the PRISCUS list
}

\author{
Katharina Pohl-Dernick ${ }^{1 *}$, Florian Meier ${ }^{1}$, Renke Maas $^{2}$, Oliver Schöffski ${ }^{1}$ and Martin Emmert ${ }^{3}$
}

\begin{abstract}
Background: Several lists of potentially inappropriate medication (PIM) for elderly patients have been developed worldwide in recent years. Those lists intend to reduce prescriptions of drugs that carry an unnecessarily high risk of adverse drug events in elderly patients. In 2010, an expert panel published the PRISCUS list for the German drug market. This study calculates the amount of drug reimbursement for PIM in Germany and potential cost effects from the perspective of statutory health insurance when these are replaced by the substitutes recommended by the PRISCUS list.

Methods: Register-based data for the 30 top-selling drugs on the PRISCUS list in 2009 for patients greater than or equal to 65 years of age were provided by the Scientific Institute of the German Local Health Care Fund. We calculated the percentage of sales and defined daily doses for patients greater than or equal to 65 years of age compared with the total statutory health insurance population. Reimbursement costs for the recommended substitutions were estimated by considering different scenarios.
\end{abstract}

Results: In 2009, drug reimbursement for the 30 top-selling PIM prescribed to patients greater than or equal to 65 years of age were calculated to be $€ 305.7$ million. Prescribing the recommended substitution medication instead of PIM would lead to an increased total reimbursement cost for the German health care system ranging between from €325.9 million to $€ 810.0$ million.

Conclusions: The results show that the substitution of PIM by medication deemed to be more appropriate for the elderly comes along with additional costs. Consequently, there is no short-term incentive for doing so from a payer perspective. Future studies have to consider the long-term effects and other sectors.

Keywords: Potentially inappropriate medication, Elderly, Drug Reimbursement, PRISCUS, Reimbursement costs

\section{Background}

Potentially inappropriate medication (PIM) can influence the safety of elderly patients' drug therapy. Several international studies have shown that PIM might lead to higher morbidity and mortality as well as it contributes to adverse drug events (ADE) and hospital admissions [1-3]. Consequently, PIM might have an impact on the health and quality of life of elderly people. Thus, a need has emerged for the systematic identification and description of inadequate pharmacotherapy for older

\footnotetext{
* Correspondence: katharina.pohl-dernick@fau.de

${ }^{1}$ Chair of Health Management, Institute of Management (IFM),

Friedrich-Alexander-Universität Erlangen-Nürnberg (FAU), Lange Gasse 20,

90403 Nuremberg, Germany

Full list of author information is available at the end of the article
}

patients. Different classification systems of critical drugs have been developed in many countries to support physicians in clinical practice such as the Beers criteria in the US [4], McLeod in Canada [5], and Laroche in France [6]. The prevalence of PIM is calculated between $13 \%$ and $43 \%$, depending on the tool used and the study setting $[7,8]$.

In Germany, the Federal Ministry of Health initiated an evaluation of PIM in 2008/2009 considering the countryspecific characteristics of the pharmaceutical market. Details of the development process have been described elsewhere [9]. The result was the so-called PRISCUS list, which comprises 83 agents classified as PIM. Altogether, 64 agents are listed on one or more international PIM lists, 12 agents are only available in Germany and seven 
are solely classified as PIM in Germany [9]. In contrast to former frameworks, the PRISCUS list is more than a listing of critical agents for elderly patients. Additional information about the adequate substitution of PIM is provided $[9,10]$. Several studies evaluate PIM in Germany and have determined the prevalence to be between $18 \%$ and $43 \%$, depending on the setting and patient group [11-14]. However, the social and economic burden has not been addressed so far.

The aim of this investigation was to analyse the reimbursement costs of PIM according to the PRISCUS list and to evaluate the economic effects of a recommended substitution from the perspective of SHI in Germany. As the PRISCUS list was explicitly developed for the German pharmaceutical market, we will use it to detect PIM in the German SHI population. We provide information about (a) the proportion of agents on the PRISCUS list prescribed to elderly people $\geq 65$ years of age, (b) the reimbursement costs of PIM from the perspective of SHI and (c) the potential costs of the recommended substitution medication.

\section{Methods}

The methodology of the study consists of three components. First, anonymised medication data on patients $\geq 65$ years of age were obtained and enriched with information about the entire SHI population. Second, costs for the prescription of surrogates were calculated based on different scenarios. Third, we conducted a literature research to identify international studies for comparison with our results.

\section{Data source}

The underlying anonymised aggregated prescription data were provided by the Scientific Institute (WIdO) of the German Local Health Care Fund (AOK). It was founded in 1976 and has the function to carry out research projects aimed at ensuring high quality and economic health care for the community of the Local Health Care Funds [15]. The WIdO has a prescription register of all drugs prescribed to patients in German SHI, excluding drugs dispensed at hospitals, over-the-counter (OTC) compounds, and prescriptions of private health insurance. Our study material is a register extraction of PRISCUS agents solely given to patients aged $\geq 65$ years in 2009 . The WIdO provided the sales volume, number of prescriptions, and the prescribed defined daily dose (DDD) for the top 30 PRISCUS agents with the highest sales volume. Additionally, the total sales volume, the total number of prescriptions, and the total amount of DDDs for all 83 agents on the PRISCUS list for the patient group $\geq 65$ years of age were provided.

The data set was enhanced with additional information from the pharmaceutical prescription report from 2010
(PPR) [16]. The PPR is an annual publication by the WIdO about the 3000 most frequently prescribed drugs to the SHI population in Germany. Therefore, the provided data set and the enriched data have the same origin. First, we extracted the sales volume, prescriptions, DDDs, and costs per DDD of all PRISCUS agents for the entire SHI population from the PPR. Second, based on the enhanced data set, we calculated the share of patients $\geq 65$ years of age in the entire SHI population for sales volume, prescriptions, and DDDs.

\section{Scenarios}

To calculate the potential costs of the recommended drug substitutions, we first derived the costs per DDD for the recommended surrogates from the PPR. In the first case, if a specifically drug was named, the costs per DDD were directly taken from the PPR. In the second case, if a drug group was recommended as an adequate alternative, a weighted average based on the costs per DDD and the prescribed annual DDDs was calculated. For example, the recommended alternatives on the PRISCUS list for indometacin are (1) paracetamol, (2) weak opioids (tramadol and codeine), (3) metamizol (after careful consideration of the risks and the benefits) and (4) "weaker" NSAIDs (e.g., ibuprofen) [9]. For (1) paracetamol and (3) metamizol, the costs per DDD were taken from the PPR (€0.49/DDD and $€ 1.51 / D D D)$. For (2) weak opioids, tramadol and codeine are explicitly recommended. Thus, the costs per DDD for these two agents were also taken from the PPR (€1.19/DDD and €2.65/ DDD). By contrast, for (4) "weaker" NSAIDs, ibuprofen is only one example of a possible substitution. Thus, the costs per DDD for the whole group of "weaker" NSAIDs were calculated (€0.56/DDD).

In a second step, the reimbursement costs for an adjusted drug therapy was estimated under the consideration of the recommended surrogate according to the PRISCUS list. We assumed that the amount of DDDs for the recommended surrogates would not differ from the actual prescribed DDDs of PIM. To calculate the economic impact, the costs per DDD for the proposed substitution were multiplied by the prescribed amount of DDDs for patients $\geq 65$ years of age. As several drugs or drug groups are recommended in some cases, a range of costs per DDD for surrogates had to be considered. Thus, we performed three different scenarios. Besides the cheapest (Scenario 1) and the most expensive surrogate (Scenario 2), we also calculated the mean costs per DDD of the proposed alternatives (Scenario 3) and the corresponding costs of the prescription.

\section{Literature review}

Two systematic literature reviews were conducted to compare the results with national and international 
studies. The first one consisted of studies dealing with the prevalence of PIM in Germany measured by applying the PRISCUS list. The second one focused on international studies analysing reimbursement costs. We carried out two Medline searches on the $2^{\text {nd }}$ of November 2014. In order to identify the prevalence of PIM in Germany, we combined 18 synonyms for PIM, such as potentially inappropriate medication, inappropriate prescribing, and inappropriate practice in prescribing with "PRISCUS". For the identification of studies of the costs of PIM, we combined the keywords cost OR costs OR cost analysis OR cost analyses OR drug reimbursement OR drug reimbursements OR economic burden OR economic evaluation and elder* OR old* OR geriatric* OR senior OR aged OR aging with the same words on PIM as in the search on prevalence. The complete search history for both searches can be obtained from the first author upon request. The search was not limited with regard to language and timeframe. Identified studies were searched by reviewing the references as well. For search 1, only studies of the prevalence of PIM in Germany were included. For search 2, papers were included if they examined the nationwide costs of PIM on an annual basis. Papers only examining the prevalence or the applicability of tools to identify PIM were excluded.

\section{Results}

In 2009, the total expenditure for all 83 agents on the PRISCUS list was €387.8 million and the reimbursement costs of the 30 top-selling agents amounted to $€ 305.7$ million $(78.8 \%)$. The highest reimbursement costs were caused by solifenacin, with $€ 32.5$ million, followed by etoricoxib ( $€ 30.6$ million), and zopiclone ( $€ 21.5$ million), as displayed in Table 1. Those three agents accounted for approximately $20 \%$ of the total sales volume. Compared with the entire SHI population in Germany, the total sales for the 30 top-selling agents were $€ 505.5$ million and $€ 562.7$ million for all 83 agents PRISCUS agents, respectively. Thus, $54.3 \%$ and $37.4 \%$ of sales can be assigned to patients $\geq 65$ years of age.

Focusing on the number of PIM prescriptions to the elderly, there were 13.75 million prescriptions of the 30 top-selling PIM. For all PRISCUS agents, there were 16.93 million prescriptions to the elderly in 2009. Compared with the entire SHI population with 26.15 million prescriptions of the 30 top-selling PRISCUS agents and 31.71 million prescriptions of all 83 PRISCUS agents, $52.6 \%$ and $44.9 \%$ of the prescriptions were allocated to the elderly, respectively. Regarding all prescriptions to the entire SHI population (626.3 million in 2009), the elderly obtained $56 \%$ (350.7 million) of them [16]. Consequently, the number of PIM prescriptions to the elderly compared with the total prescriptions to patients $\geq 65$ years of age was $4.8 \%$.
The sum of DDDs for the 30 top-selling PRISCUS agents was 502.9 million DDDs and 598.3 million DDDs for all 83 agents. The leading agents were doxazosin (51.4 million DDDs), amitriptyline (45.7 million DDDs), and sotalol (34.0 million DDDs). In the entire SHI population, 864.40 million DDDs of the 30 top-selling PIM and 1,124.0 million DDDs of all PIM were prescribed. Thus, the majority of prescribed DDDs of the 30 topselling PIM (58.2 \%) may be assigned to patients for whom the drugs are potentially inadequate. Regarding all PIM on the PRISCUS list, $53.3 \%$ of the DDDs for the entire SHI population were prescribed to the elderly.

Table 2 shows information on the costs and DDDs for PIM and the recommended surrogates on the PRISCUS list. When applying Scenario 1, the reimbursement costs for the 30 top-selling agents on the PRISCUS list would be $€ 325.93$ million and this would lead to higher total costs of $€ 20.23$ million ( $+6.6 \%)$. For Scenario 2, the costs of medication would rise to $€ 810.03$ million and cause additional costs of $€ 504.33$ million (+165.0 \%). By applying Scenario 3, the costs for the 30 top-selling drugs would rise by $€ 124.76$ million to $€ 430.46$ million (+40.8\%).

Considering the results for Scenario 1, only 10 surrogates lead to increased reimbursement costs, whereas the substitution of the remaining 20 agents leads to a cost decrease. However, these 10 surrogates increase the costs by $+200.0 \%$ on average, whereas the decrease is $-53.3 \%$ on average. The cost driver in Scenario 1 is memantine (€3.86/DDD), a proposed surrogate for piracetam (€0.45/DDD), pentoxifyllin ( $€ 0.54 / \mathrm{DDD})$, and naftidrofuryl ( $€ 1.11 / D D D)$. The substitution of these three agents by memantine causes costs of $€ 70.6$ million, $€ 57.9$ million and $€ 29.0$ million, respectively. This results in a potential cost increase of $€ 133.9$ million ( $+467.0 \%)$, from $€ 23.6$ million to $€ 157.5$ million.

In Scenario 2, 22 agents increase the costs by $+289.0 \%$ on average. The most cost-intensive surrogates are acetycholinesterase inhibitors (€4.25/DDD) - as proposed alternatives for piracetam, pentoxifyllin, and naftidrofuryl - and this would lead to increased reimbursement costs of $€ 173.4$ million (+635.0 \%), from $€ 23.6$ million to $€ 197.0$ million. The other cost drivers in Scenario 2 are the recommended substitutes for etoricoxib (e.g., lamotrigine, oxcarbazepine, gabapentin). This substitution would raise reimbursement costs from $€ 30.6$ million to $€ 101.56$ million $(+232.0 \%)$. If indomethacin $(0.46 € / \mathrm{DDD})$ was substituted by codeine $(2.65 € / \mathrm{DDD})$, the cost of the medication would rise by $+476.4 \%$. Only the substitution of eight agents would lead to a decrease in total reimbursement costs, by $-27.5 \%$.

Considering the mean surrogate costs in Scenario 3, in 14 cases the substitution of the 30 top-selling agents would lead to a decrease in reimbursement costs by $-37.9 \%$ on 
Table 130 top-selling agents on PRISCUS list (Prices in €, Reference year 2009)

\begin{tabular}{|c|c|c|c|c|c|c|c|c|c|c|c|c|c|}
\hline \multirow[b]{2}{*}{ No. } & \multirow[b]{2}{*}{ ATC-Code } & \multirow[b]{2}{*}{ Agent } & \multicolumn{4}{|c|}{ Aged 65 and above } & \multicolumn{4}{|c|}{ Entire SHI population } & \multicolumn{3}{|c|}{ Ratio aged $\geq 65$ and entire $\mathrm{SHI}$} \\
\hline & & & $\begin{array}{l}\text { DDD (in Mio.) } \\
\geq 65 \text { years }\end{array}$ & $\begin{array}{l}\text { Prescriptions } \\
\geq 65 \text { (in Mio.) }\end{array}$ & $\begin{array}{l}\text { Sales } \geq 65 \\
\text { (in Mio. €) }\end{array}$ & $\begin{array}{l}\text { Costs per } \\
\text { DDD } \geq 65\end{array}$ & $\begin{array}{l}\text { Total DDD } \\
\text { (in Mio) }\end{array}$ & $\begin{array}{l}\text { Total prescriptions } \\
\text { (in Mio) }\end{array}$ & $\begin{array}{l}\text { Total sales } \\
\text { (in Mio. €) }\end{array}$ & Costs per DDD & DDD & Prescriptions & Sales \\
\hline 1 & C02CA04 & Doxazosin & 51.4 & 0.56 & 19.5 & $0.38 €$ & 60.2 & 0.81 & 28.1 & $0.38 €$ & $85.38 \%$ & $69.47 \%$ & $69.37 \%$ \\
\hline 2 & N06AA09 & Amitriptyline & 45.7 & 1.13 & 19.8 & $0.43 €$ & 94.5 & 2.28 & 40.5 & $0.43 €$ & $48.36 \%$ & $49.66 \%$ & $48.88 \%$ \\
\hline 3 & C07AA07 & Sotalol & 34.0 & 0.53 & 10.2 & $0.30 €$ & 36.6 & 0.68 & 13.0 & $0.30 €$ & $92.90 \%$ & $78.39 \%$ & $78.56 \%$ \\
\hline 4 & C01AA02 & Acetyldigoxin & 32.5 & 0.93 & 11.7 & $0.36 €$ & 36.4 & 1.05 & 13.3 & $0.36 €$ & $89.29 \%$ & $88.62 \%$ & $88.06 \%$ \\
\hline 5 & N05CF01 & Zopiclon $^{\mathrm{a}}$ & 28.0 & 1.44 & 21.5 & $0.77 €$ & 47.3 & 2.62 & 38.7 & $0.79 €$ & $59.20 \%$ & $54.87 \%$ & $55.56 \%$ \\
\hline 6 & M01AH05 & Etoricoxib & 25.2 & 0.50 & 30.6 & $1.21 €$ & 51.7 & 1.07 & 62.4 & $1.21 €$ & $48.74 \%$ & $46.61 \%$ & $49.04 \%$ \\
\hline 7 & N06AA12 & Doxepin & 24.2 & 0.77 & 13.1 & $0.54 €$ & 54.2 & 1.68 & 29.1 & $0.52 €$ & $44.65 \%$ & $45.71 \%$ & $45.07 \%$ \\
\hline 8 & G04BD08 & Solifenacin & 22.6 & 0.24 & 32.5 & $1.44 €$ & 31.3 & 0.33 & 44.8 & $1.43 €$ & $72.20 \%$ & $72.84 \%$ & $72.54 \%$ \\
\hline 9 & N05CF02 & Zolpidem ${ }^{a}$ & 21.3 & 1.09 & 16.3 & $0.77 €$ & 32.5 & 1.81 & 26.7 & $0.78 €$ & $65.54 \%$ & $60.36 \%$ & $61.14 \%$ \\
\hline 10 & N06BX03 & Piracetam & 18.3 & 0.41 & 8.2 & $0.45 €$ & 20.6 & 0.48 & 9.7 & $0.45 €$ & $88.83 \%$ & $85.85 \%$ & $84.86 \%$ \\
\hline 11 & C04AD03 & Pentoxifylline & 15.0 & 0.35 & 7.2 & $0.48 €$ & 17.7 & 0.57 & 11.0 & $0.54 €$ & $84.75 \%$ & $61.29 \%$ & $65.61 \%$ \\
\hline 12 & N05BA08 & Bromazepam & 14.9 & 0.66 & 8.6 & $0.58 €$ & 21.2 & 1.04 & 13.5 & $0.60 €$ & $70.28 \%$ & $63.40 \%$ & $63.65 \%$ \\
\hline 13 & N06AA06 & Trimipramine & 14.6 & 0.49 & 10.8 & $0.74 €$ & 31.6 & 1.12 & 24.5 & $0.73 €$ & $46.20 \%$ & $43.66 \%$ & $44.17 \%$ \\
\hline 14 & N05BA01 & Diazepam & 13.1 & 0.53 & 6.0 & $0.46 €$ & 31.0 & 1.30 & 15.3 & $0.47 €$ & $42.26 \%$ & $40.71 \%$ & $39.16 \%$ \\
\hline 15 & N05CD06 & Lormetazepam $^{a}$ & 12.5 & 0.38 & 5.2 & $0.42 €$ & 15.3 & 0.51 & 7.0 & $0.42 €$ & $81.70 \%$ & $74.50 \%$ & $74.20 \%$ \\
\hline 16 & C02CA08 & Terazosin & 12.3 & 0.19 & 6.6 & $0.54 €$ & 8.0 & 0.24 & 8.2 & $0.56 €$ & $-\mathrm{b}$ & - & - \\
\hline 17 & C02AC01 & Clonidine & 11.7 & 0.33 & 5.9 & $0.50 €$ & 17.7 & 0.71 & 13.5 & $0.34 €$ & $66.10 \%$ & $46.72 \%$ & $43.86 \%$ \\
\hline 18 & C02LA01 & $\begin{array}{l}\text { Reserpine and } \\
\text { Diuretics }\end{array}$ & 11.3 & 0.12 & 4.7 & $0.42 €$ & 13.1 & 0.14 & 5.5 & $0.42 €$ & $86.26 \%$ & $88.82 \%$ & $85.45 \%$ \\
\hline 19 & $\mathrm{C01BC04}$ & Flecainide & 9.7 & 0.22 & 11.4 & $1.18 €$ & 14.1 & 0.34 & 17.5 & $1.17 €$ & $68.79 \%$ & $64.05 \%$ & $65.30 \%$ \\
\hline 20 & G04BD04 & Oxybutynin & 9.3 & 0.30 & 8.8 & $0.95 €$ & 11.3 & 0.45 & 15.1 & $0.95 €$ & $82.30 \%$ & $66.64 \%$ & $58.14 \%$ \\
\hline 21 & M01AC06 & Meloxicam & 9.3 & 0.21 & 3.3 & $0.35 €$ & 14.8 & 0.41 & 6.3 & $0.38 €$ & $62.84 \%$ & $50.66 \%$ & $52.12 \%$ \\
\hline 22 & N06AB03 & Fluoxetine & 8.8 & 0.10 & 2.9 & $0.33 €$ & 43.3 & 0.58 & 17.3 & $0.32 €$ & $20.32 \%$ & $17.32 \%$ & $16.79 \%$ \\
\hline 23 & M01AB01 & Indometacin & 8.7 & 0.25 & 4.0 & $0.46 €$ & 14.2 & 0.53 & 8.6 & $0.48 €$ & $61.27 \%$ & $47.36 \%$ & $46.63 \%$ \\
\hline 24 & C01AA08 & Metildigoxin & 8.4 & 0.17 & 2.7 & $0.32 €$ & 9.5 & 0.20 & 3.0 & $0.32 €$ & $88.42 \%$ & $86.65 \%$ & $90.00 \%$ \\
\hline 25 & М03ВX07 & Tetrazepam & 8.3 & 0.57 & 8.0 & $0.96 €$ & 22.0 & 2.08 & 26.8 & $1.19 €$ & $37.73 \%$ & $27.46 \%$ & $29.90 \%$ \\
\hline 26 & C04AX21 & Naftidrofuryl & 7.5 & 0.34 & 8.2 & $1.09 €$ & 9.0 & 0.43 & 10.2 & $1.11 €$ & 83.33 \% & $78.49 \%$ & $80.17 \%$ \\
\hline 27 & C08CA05 & Nifedipine ${ }^{a}$ & 6.5 & 0.26 & 3.8 & $0.58 €$ & 69.7 & 1.32 & 22.2 & $0.29 €$ & $9.33 \%$ & $19.70 \%$ & $17.11 \%$ \\
\hline 28 & M01AB11 & Acemetacin & 6.4 & 0.16 & 4.9 & $0.77 €$ & 9.8 & 0.28 & 8.3 & $0.80 €$ & $65.31 \%$ & $56.34 \%$ & $58.76 \%$ \\
\hline 29 & М03ВХ01 & Baclofen & 6.0 & 0.26 & 5.2 & $0.87 €$ & 17.1 & 0.66 & 16.1 & $0.93 €$ & $35.09 \%$ & $39.12 \%$ & $32.31 \%$ \\
\hline
\end{tabular}


Table 130 top-selling agents on PRISCUS list (Prices in €, Reference year 2009) (Continued)

\begin{tabular}{|c|c|c|c|c|c|c|c|c|c|c|c|c|c|}
\hline 30 & J01XE01 & Nitrofurantoin & 5.4 & 0.26 & 4.1 & $0.76 €$ & 8.7 & 0.43 & 6.7 & $0.85 €$ & $62.07 \%$ & $59.99 \%$ & $61.45 \%$ \\
\hline Sum Top30 & & & 502.9 & 13.75 & 305.7 & & 864.40 & 26.15 & 562.71 & & $58.18 \%$ & $52.59 \%$ & $54.33 \%$ \\
\hline Sum all 83 agents & & & 598.7 & 16.93 & 387.8 & & $1,124.00$ & 37.71 & $1,037.37$ & & $53.27 \%$ & $44.89 \%$ & $37.38 \%$ \\
\hline
\end{tabular}

aPRISCUS agents which are potentially inappropriate at certain dose or release form

${ }^{\mathrm{b}}$ The PPR does include the 3,000 top-selling drugs. Several traded products with terazosin as agent are sold less frequently and therefore not included. The published amount of DDD of terazosin is lower than the share of DDD taken by the elderlies. Consequently, calculation is misleading 
Table 2 Costs of surrogates for the elderly (Prices in $€$, Reference year 2009)

\begin{tabular}{|c|c|c|c|c|c|c|c|c|c|c|c|c|}
\hline \multirow[t]{2}{*}{ ATC-Code } & \multirow[t]{2}{*}{ Agent } & \multirow[t]{2}{*}{$\begin{array}{l}\text { Costs per } \\
\text { DDD in } €\end{array}$} & \multirow[t]{2}{*}{$\begin{array}{l}\mathrm{DDD} \\
\text { (in Mio.) }\end{array}$} & \multirow[t]{2}{*}{$\begin{array}{l}\text { Surrogates according to } \\
\text { PRISCUS list }\end{array}$} & \multicolumn{5}{|c|}{$\begin{array}{l}\text { Costs per DDD of } \\
\text { surrogates in } €\end{array}$} & \multicolumn{3}{|c|}{$\begin{array}{l}\text { Total costs of } \\
\text { surrogates in Mio. } €\end{array}$} \\
\hline & & & & & Min & & Max & & Mean & Min & Max & Mean \\
\hline C02CA04 & Doxazosin & 0.38 & 51.4 & $\begin{array}{l}\text { Other antihypertensives: ACE inhibitors, } \\
\text { angiotensin receptor blockers, diuretics, } \\
\text { beta blocker, calcium channel blocker }\end{array}$ & ACE inhibitors & 0.13 & $\begin{array}{l}\text { Angiotensin } \\
\text { receptor blockers }\end{array}$ & 1.08 & 0.23 & 6.64 & 55.51 & 23.73 \\
\hline N06AA09 & Amitriptyline & 0.43 & 45.7 & SSRIs, mirtazapine & SSRIS & 0.49 & Mirtazapine & 0.69 & 0.53 & 22.39 & 31.53 & 23.32 \\
\hline C07AA07 & Sotalol & 0.30 & 34.0 & Beta blocker, amiodarone, propafenone & beta blocker & 0.30 & Amiodarone & 0.72 & 0.77 & 10.09 & 24.48 & 21.69 \\
\hline C01AA02 & Acetyldigoxin & 0.36 & 32.5 & Beta blocker, diuretics, ACE inhibitors & ACE inhibitors & 0.13 & Beta blocker & 0.30 & 0.18 & 4.20 & 9.65 & 24.43 \\
\hline N05CF01 & Zopiclon $^{\mathrm{a}}$ & 0.77 & 28.0 & $\begin{array}{l}\text { Valerian, sedative anti-depressants } \\
\text { (trazodone, mianserin, mirtazapine), } \\
\text { opipramol, low-potency neuroleptics } \\
\text { (melperone \& pipamperone) }\end{array}$ & Valerian & 0.00 & $\begin{array}{l}\text { Low-potency } \\
\text { neuroleptics }\end{array}$ & 2.32 & 0.77 & 0.00 & 64.86 & 11.80 \\
\hline M01AH05 & Etoricoxib & 1.21 & 25.2 & $\begin{array}{l}\text { Paracetamol, weak opioids (codeine, } \\
\text { tramadol), Metamizole, weak NSAID, } \\
\text { antidepressants (long-acting tranquilizers, } \\
\text { tricyclic antidepressants, other non- } \\
\text { serotomergic monoamine oxidase } \\
\text { inhibitors, SSRIs, other Selective reuptake } \\
\text { inhibitors, other antidepressants), } \\
\text { anticonvulsants (older anti-epileptics, } \\
\text { newer anti-epileptics, neuromuscular } \\
\text { blocking agents) }\end{array}$ & Paracetamol & 0.49 & $\begin{array}{l}\text { Newer anti- } \\
\text { epileptics }\end{array}$ & 4.03 & 3.90 & 12.35 & 101.56 & 16.50 \\
\hline N06AA12 & Doxepin & 0.54 & 24.2 & SSRIs, mirtazapine & SSRIS & 0.49 & Mirtazapine & 0.69 & 0.53 & 11.86 & 16.70 & 12.94 \\
\hline G04BD08 & Solifenacin & 1.44 & 22.6 & Trospium & Trospium & 1.05 & Trospium & 1.05 & 1.05 & 23.73 & 23.73 & 5.89 \\
\hline N05CF02 & Zolpidema & 0.77 & 21.3 & $\begin{array}{l}\text { Valerian, sedative anti-depressants } \\
\text { (trazodone, mianserin, mirtazapine), } \\
\text { opipramol, low-potency neuroleptics } \\
\text { (melperone \& pipamperone) }\end{array}$ & Valerian & 0.00 & $\begin{array}{l}\text { Low-potency } \\
\text { neuroleptics }\end{array}$ & 2.32 & 0.77 & 0.00 & 49.34 & 2.95 \\
\hline N06BX03 & Piracetam & 0.45 & 18.3 & $\begin{array}{l}\text { ACE inhibitors (donepezil, galantamine, } \\
\text { rivastigmine), Memantine }\end{array}$ & Memantine & 3.86 & $\begin{array}{l}\text { Acetylcholinesterase } \\
\text { inhibitors }\end{array}$ & 4.25 & 4.11 & 70.64 & 77.78 & 7.80 \\
\hline C04AD03 & Pentoxifylline & 0.48 & 15.0 & $\begin{array}{l}\text { ACE inhibitors (donepezil, galantamine, } \\
\text { rivastigmine), Memantine }\end{array}$ & Memantine & 3.86 & Acetylcholinesterase inhibitors & 4.25 & 4.11 & 57.90 & 63.75 & 10.36 \\
\hline N05BA08 & Bromazepam & 0.58 & 14.9 & $\begin{array}{l}\text { Lorazepam, lormetazepam, short-acting } \\
\text { benzodiazepine \& benzodiazepine } \\
\text { receptor agonists (zolpidem, zopiclone, } \\
\text { brotizolam, triazolam, zaleplon), } \\
\text { opipramol, mirtazapine, low-potency } \\
\text { neuroleptics (melperone \& pipamperone) }\end{array}$ & Lormetazepam & 0.42 & $\begin{array}{l}\text { Low-potency } \\
\text { neuroleptics }\end{array}$ & 2.32 & 0.75 & 6.26 & 34.52 & 9.77 \\
\hline N06AA06 & Trimipramine & 0.74 & 14.6 & SSRIs, mirtazapine & SSRI & 0.49 & Mirtazapine & 0.69 & 00.53 & 7.15 & 10.07 & 11.23 \\
\hline N05BA01 & Diazepam & 0.46 & 13.1 & $\begin{array}{l}\text { Lorazepam, lormetazepam, short-acting } \\
\text { benzodiazepine \& benzodiazepine } \\
\text { receptor agonists (zolpidem, zopiclone, } \\
\text { brotizolam, triazolam, zaleplon), opipramol, }\end{array}$ & Lormetazepam & 0.42 & $\begin{array}{l}\text { Low-potency } \\
\text { neuroleptics }\end{array}$ & 2.32 & 0.75 & 5.50 & 30.35 & 75.19 \\
\hline
\end{tabular}


Table 2 Costs of surrogates for the elderly (Prices in $€$, Reference year 2009) (Continued)

\begin{tabular}{|c|c|c|c|c|c|c|c|c|c|c|c|c|}
\hline & & & & $\begin{array}{l}\text { mirtazapine, low-potency neuroleptics } \\
\text { (melperone \& pipamperone) }\end{array}$ & & & & & & & & \\
\hline N05CD06 & Lormetazepam $^{\text {a }}$ & 0.42 & 12.5 & $\begin{array}{l}\text { Valerian, sedative anti-depressants } \\
\text { (trazodone, mianserin, mirtazapine), } \\
\text { opipramol, zolpidem, low-potency } \\
\text { neuroleptics (melperone \& pipamperone) }\end{array}$ & Valerian & 0.00 & $\begin{array}{l}\text { Low-potency } \\
\text { neuroleptics }\end{array}$ & 2.32 & 0.78 & 0.00 & 28.96 & 30.82 \\
\hline C02CA08 & Terazosin & 0.54 & 12.3 & $\begin{array}{l}\text { Other antihypertensives: ACE inhibitors } \\
\text { angiotensin receptor blockers, diuretics, } \\
\text { beta blocker, calcium channel blocker } \\
\text { (except nifedipine) }\end{array}$ & ACE inhibitors & 0.13 & Beta blocker & 0.30 & 0.18 & 1.59 & 3.65 & 6.79 \\
\hline C02AC01 & Clonidine & 0.50 & 11.7 & $\begin{array}{l}\text { Other antihypertensives: ACE inhibitors, } \\
\text { angiotensin receptor blockers, diuretics, } \\
\text { beta blocker , calcium channel blocker }\end{array}$ & ACE inhibitors & 0.13 & $\begin{array}{l}\text { Angiotensin receptor } \\
\text { blockers }\end{array}$ & 1.08 & 0.23 & 1.51 & 12.64 & 61.64 \\
\hline C02LA01 & $\begin{array}{l}\text { Reserpine and } \\
\text { Diuretics }\end{array}$ & 0.42 & 11.3 & $\begin{array}{l}\text { Other antihypertensives: ACE inhibitors, } \\
\text { angiotensin receptor blockers, diuretics, } \\
\text { beta blocker, calcium channel blocker } \\
\text { (except nifedipine) }\end{array}$ & ACE inhibitors & 0.13 & Beta blocker & 0.30 & 0.18 & 1.46 & 3.35 & 2.24 \\
\hline $\mathrm{C} 01 \mathrm{BC} 04$ & Flecainide & 1.18 & 9.7 & Beta blocker, amiodarone & Beta blocker & 0.30 & Amiodarone & 0.72 & 0.30 & 2.88 & 6.98 & 9.87 \\
\hline G04BD04 & Oxybutynin & 0.95 & 9.3 & Trospium & Trospium & 1.05 & Trospium & 1.05 & 1.05 & 9.77 & 9.77 & 2.69 \\
\hline M01AC06 & Meloxicam & 0.35 & 9.3 & $\begin{array}{l}\text { Paracetamol, weak opioids (codeine, } \\
\text { tramadol), metamizole, "weaker" NSAID }\end{array}$ & Paracetamol & 0.49 & Codeine & 2.65 & 0.72 & 4.56 & 24.65 & 9.69 \\
\hline N06AB03 & Fluoxetine & 0.33 & 8.8 & $\begin{array}{l}\text { Other SSRIs (citalopram, paroxetine, } \\
\text { escitalopram, sertraline, escitalopram), } \\
\text { trazodone, mitrazapine }\end{array}$ & other SSRIs & 0.51 & Trazodone & 1.44 & 0.56 & 4.50 & 12.67 & 8.71 \\
\hline M01AB01 & Indometacin & 0.46 & 8.7 & $\begin{array}{l}\text { Paracetamol, weak opioids (codeine, } \\
\text { tramadol), metamizole, "weaker" NSAID }\end{array}$ & Paracetamol & 0.49 & Codeine & 2.65 & 0.72 & 4.26 & 23.06 & 4.60 \\
\hline C01AA08 & Metildigoxin & 0.32 & 8.4 & Beta blocker, diuretics & Diuretics & 0.21 & Beta blocker & 0.30 & 0.26 & 1.76 & 2.49 & 2.06 \\
\hline M03ВХ07 & Tetrazepam & 0.96 & 8.3 & $\begin{array}{l}\text { Tolperisone, short-acting benzodiazepine } \\
\text { \& benzodiazepine receptor agonists } \\
\text { (zolpidem, zopiclone, brotizolam, triazolam, } \\
\text { zaleplon), intermediate-acting tranquilizers } \\
\text { (bromazepan, oxazepam, lorazepam, } \\
\text { alprazolam, buspirone) }\end{array}$ & $\begin{array}{l}\text { intermediate-acting } \\
\text { Tranquilizers }\end{array}$ & 0.71 & Tolperisone & 1.55 & 0.82 & 5.89 & 12.87 & 12.59 \\
\hline C04AX21 & Naftidrofuryl & 1.09 & 7.5 & $\begin{array}{l}\text { ACE inhibitors (donepezil, galantamine, } \\
\text { rivastigmine), memantine }\end{array}$ & Memantine & 3.86 & $\begin{array}{l}\text { Acetylcholinesterase } \\
\text { inhibitors }\end{array}$ & 4.25 & 4.11 & 28.95 & 31.88 & 6.25 \\
\hline C08CA05 & Nifedipine $^{a}$ & 0.58 & 6.5 & $\begin{array}{l}\text { Other antihypertensives: ACE inhibitors, } \\
\text { angiotensin receptor blockers, diuretics, } \\
\text { beta blocker, calcium channel blocker } \\
\text { (except nifedipine) }\end{array}$ & ACE inhibitors & 0.13 & Beta blocker & 0.30 & 0.18 & 0.84 & 1.93 & 1.19 \\
\hline M01AB11 & Acemetacin & 0.77 & 6.4 & $\begin{array}{l}\text { Paracetamol, weak opioids (codeine, } \\
\text { tramadol), metamizole, "weaker" NSAID }\end{array}$ & Paracetamol & 0.49 & Codeine & 2.65 & 0.72 & 3.14 & 16.96 & 6.68 \\
\hline
\end{tabular}


Table 2 Costs of surrogates for the elderly (Prices in $€$, Reference year 2009) (Continued)

\begin{tabular}{|c|c|c|c|c|c|c|c|c|c|c|c|c|}
\hline M03BX01 & Baclofen & 0.87 & 6.0 & Tolperisone, tizanidine & Tizanidine & 1.09 & Tolperisone & 1.55 & 1.45 & 6.54 & 9.30 & 4.91 \\
\hline J01XE01 & Nitrofurantoin & 0.76 & 5.4 & $\begin{array}{l}\text { Other antibiotics in accordance with } \\
\text { antibiogramm: cephalosporins, } \\
\text { cotrimoxazole, trimethoprim }\end{array}$ & Cotrimoxazole & 1.77 & Trimethoprim & 2.79 & 2.33 & 9.56 & 15.07 & 2.16 \\
\hline \multicolumn{3}{|c|}{ Sum Top 30} & 502.9 & & & & & & & 325.93 & 810.03 & 430.46 \\
\hline
\end{tabular}

aPRISCUS agents which are potentially inappropriate at certain dose or release form 
average. However, these savings would be compensated for by the 16 remaining agents, which lead to an average increase of $+160.7 \%$.

\section{Discussion}

To our knowledge, this is the first study to assess the reimbursement costs of PIM according to the PRISCUS list in Germany from a SHI perspective. We conducted a comprehensive analysis based on prescription data from 2009 in order to depict the prevalence of PIM in the elderly in Germany and to quantify the economic impact of prescriptions as well as the substitution costs for the recommended medication.

\section{The prevalence of PIM in the elderly}

Our search yielded eight studies dealing with the prevalence of PIM in Germany, according to the PRISCUS list $[12-14,17-21]$. The present study is based on prescription data in the ambulant stetting for all patients $\geq 65$ years of age in German SHI, and results in a prescription prevalence of $4.8 \%$ in 2009. The investigations of Amann et al. and Schubert et al. are most comparable regarding the database. Amann et al. calculated a standardised prevalence of $28.3 \%$ based on data on 800,000 insurees [17]. A similar study design was chosen by Schubert et al. [13]. They used claims data from German health insurance providers to calculate a prevalence of $22.0 \%$. However, they both analysed the prevalence at the patient level not at the prescription level. Therefore, the comparison is limited.

The six remaining studies calculated prevalence based on smaller patient samples and in different study settings. The results lay between $16.6 \%$ in an emergency department [14] and $43 \%$ in geriatric rehabilitation [12]. For patients in nursing homes, a prevalence of $31.2 \%$ was calculated [18], while for elderly people living at home the prevalence was lower at $18 \%$ [19], 28.4 \% [20] and $29.0 \%$ [21]. Thus, the prevalence of PIM in the elderly in Germany is between one quarter and one third, but this depends on the study setting, possible limitations concerning indication, data collection, and the consideration of the limitations of the PRISCUS list (dose dependency and release form).

\section{Economic assessment of PIM and the recommended substitution}

A search of economic analyses showed that limited information is available. In total, 12 publications dealing with the costs of PIM were identified, but none of them considered German SHI. The studies were conducted in the US [22-26], Ireland [7, 27, 28], Japan [8], Finland [29], Saudi Arabia [30], and Switzerland [31]. They used different lists and mostly calculated the costs of PIM at the patient level $[7,8,22,25]$. As our study addresses the annual economic relevance of PIM for SHI in Germany, only four studies from the US [24], Ireland
[27, 28], and Finland [29] that addressed the national perspective are eligible for the comparison.

Bradley et al. analysed the PIM of patients $>70$ years of age according to the STOPP criteria in Northern Ireland, using data from the enhanced prescribing database of the National Health Service [27]. In total, costs of $€ 6.1$ million in 2009/2010 were calculated. This equals $5.38 \%$ of total expenses on pharmaceuticals for people $>70$ years of age in Northern Ireland. Another study of Ireland by Cahir et al. published costs of $€ 45.6$ million for drug ingredients, tax, and pharmacy surcharges in 2007, representing $9 \%$ of the overall expenditure on pharmaceuticals in people aged $>70$ years [28]. The calculation applying the STOPP criteria was based on the pharmacy claims of the National Shared Services Primary Care Reimbursement Service of the Health Service Executive. In the third study, Leikola et al. calculated reimbursement costs of $€ 2.9$ million for PIM medication to people $>65$ years of age in Finland in 2007, using the criteria of Fick and Beers [29]. This represents $0.7 \%$ of total drug reimbursement for people aged $>65$ in Finland. The register-based crosssectional study used data from Finland's social insurance institution. The study from Fu et al., conducted in the US, calculated total health care expenditures of US $\$ 7.2$ billion for community-dwelling patients exposed to PIM according to the criteria of Fick and Beers [24]. For medication, about $20 \%$ of these costs were calculated. Consequently, costs of approximately US $\$ 1.44$ billion were calculated for PIM in people $>65$ years of age in 2001 in the US.

In our study, the reimbursement costs of PIM were $€ 387.8$ million. Thus, the total reimbursement costs for PIM prescriptions in Germany are significantly higher than those in Finland, Ireland, and Northern Ireland, but lower than those in the US. The total sales of all proprietary mediclinical products in SHI were $€ 28,499$ million [16]. Thus, the proportion of PIM sales to elderly patients represents $1.36 \%$. However, a comparison of this number is hardly possible, as the studies from Finland, Ireland, and Northern Ireland used sales to elderly patients as the reference figure.

In general, the comparability of our results with other international studies is limited. First, different tools were used to detect PIM: Bradley et al. and Cahir et al. applied the STOPP criteria, which contain 65 criteria systematised according to the physiological systems to identify PIM $[27,28,32]$. The studies of Leikola et al. and $\mathrm{Fu}$ et al. used the criteria of Fick and Beer from 2003, which partly consider the diagnoses or conditions of patients [24, 29, 33]. In contrast to both tools, the PRISCUS list considers neither the physiological system nor diagnoses or conditions. Second, the lists were applied to different age groups: Bradley et al. calculated costs for pharmaceutical expenses for patients $\geq 70$ years of age [27]. Our investigation covered prescriptions for 
patients $\geq 65$ years of age. Third, different perspectives on costs were used in the studies: Cahir et al. summed the net ingredient costs, the value added tax, and the pharmacist dispensing fee [28]. In our study, costs per DDD are based on the sales data of each drug. Thus, the different approaches make a comparison of costs of PIM even more difficult.

Regarding PIM substitution, reimbursement costs are about to rise, independent of the scenario. If all prescriptions of PIM were substituted by the low-cost alternative, the total costs would be $€ 325.9$ million. Substitution by the most expensive alternative would lead to a total cost of $€ 810.0$ million. This gap can be explained by the enormous difference in the costs of the recommended therapeutic alternatives. For example, there are several different alternatives for etoricoxib (e.g., paracetamol, weak opioids (codeine, tramadol), metamizol, anticonvulsants (older anti-epileptics, newer anti-epileptics, neuromuscular blocking agents)). The cheapest alternative to etoricoxib $(€ 1.21 / \mathrm{DDD})$ is paracetamol (€0.49/DDD). The most expensive alternative is newer anti-epileptics at $€ 4.03 / \mathrm{DDD}$. The difference in costs per DDD might result in enormous cost differences in each scenario. By contrast, for solifenacin, which costs $€ 1.43 / \mathrm{DDD}$ according to the PPR, the only alternative according to the PRISCUS list is trospium, with a cost of $€ 1.05 / D D D$. Consequently, in both scenarios the substitution of solifenacin will decrease the total costs.

\section{Implication for $\mathrm{SHI}$ and future research}

From a short-term economic perspective, there would be little incentive for SHI to support the substitution of PRISCUS agents. However, it has been shown that PIM leads to ADE which in turn can cause inpatient costs $[14,34,35]$. Furthermore, the application of PIM is directly associated with an increased risk of acute hospital admission [36-41]. Moreover, in many clinical cases there may be no adequate substitute for a PIM. In these cases, the discontinuation of the PIM without providing a substitute will be the best choice from a clinical as well as from an economical perspective. Thus, from a longterm perspective, the additional costs of physician consultation and hospitalisation due to ADEs caused by PIM might decrease and compensate for the costs of substituting the PRISCUS agents.

Our research revealed several aspects to be considered in future research on PIM. The PRISCUS list was published in 2010. Our study is based on data from 2009. Thus, our study does not analyse the effect of the PRISCUS list and possible changes in the prescription behaviour of physicians in Germany, but serves as a reference for future comparisons. Further research should investigate if and to what extent prescription patterns and PIM costs have developed due to the PRISCUS list in order to show the influence and relevance for practical use.
Our investigation demonstrated that PIM is a relevant topic in drug therapy in the elderly in Germany. The elderly account for approximately $55 \%$ of the sales of the 30 top-selling PRISCUS agents. Similar percentages were calculated for DDDs and prescriptions. The proportion of sales, DDDs, and prescriptions to the elderly is lower in all 83 agents of the PRISCUS list. The decrease underlines that only some of the 83 PRISCUS agents are really relevant. Thus, from an economic perspective and in terms of feasibility, a shorter PRISCUS list might be more applicable for the daily health service. However, the medical perspective has to be taken into consideration as well. A short-list should also include those agents with the worst risk/benefit ratios for the elderly.

\section{Limitations}

There are limitations to this study as a result of the methods used. First, for several PIM the recommended substitutions may not be clinically adequate in a majority of clinical cases, while in other cases the recommended substitution would lead to off-label use. For example, herbal products, such as valerian, are often named as surrogates for psycholeptics (zopiclone, zolpidem and lormetazepam). From a medical perspective, however, it is questionable if a highly effective sedative can be completely substituted by herbal alternatives. Furthermore, valerian is a non-refundable OTC and no costs occur by substituting it. Consequently, in the low-cost scenario, approximately $€ 42$ million can be "saved" by applying valerian. Second, some PRISCUS agents are only potentially inappropriate, if a certain dose is exceeded or a certain release form is applied. This was not taken into account, as the data set of WIdO consisted of total prescriptions. Third, we only analysed the 30 top-selling drugs.

Another limitation is related to our applied scenarios. In general, the scenarios assumed that a PIM would be fully substituted by the cheapest, most expensive, or mean costs. However, a complete substitution might be unlikely in daily practice, which highlights the need for a calculation based on individual patient data. In general, the proposed substitutions have to be evaluated carefully by physicians. In some cases, groups of potential surrogates include other medication form the PRISCUS list. For example, the recommended substitution of amitriptyline are SSRI. However, the group of SSRI comprises PIM as well e.g. fluoxetine. It remains unclear if in those cases PIM might be substituted by another PIM as well. For our study, we included all recommended surrogates.

\section{Conclusions}

This is the first study to assess the economic relevance of PIM on the PRISCUS list in Germany from a SHI 
perspective. It was shown that PIM is present in Germany and approximately $5 \%$ of all prescriptions made to people aged $\geq 65$ years are potentially inappropriate. Moreover, the reimbursement costs of PIM in the elderly account for $1.36 \%$ of total sales to patients $\geq 65$ years of age. The substitution of PRISCUS agents would lead to an increase in costs independent of the chosen scenario. Thus, from a short-term perspective, there is no incentive for SHI to promote substitution. However, in the long-term, hospitalisation and recurrent physician visits should be addressed.

\section{Ethics approval and consent to participate \\ Not applicable.}

\section{Consent for publication \\ Not applicable.}

\section{Availability of data and materials}

The datasets supporting the conclusions of this article are included within the article.

\section{Abbreviations}

ADE: adverse drug events; AOK: German local health care fund; DDD: defined daily dose; OTC: over-the-counter; PIM: potentially inappropriate medication; PPR: pharmaceutical prescription report; SHI: statutory health insurance; WIdO: scientific institute of the German local health care fund.

\section{Competing interests}

The authors declare that they have no competing interests.

\section{Authors' contributions}

KPD, FM and RM conceptualized and designed the study. FM and KPD were responsible for data collection and data assembly. KPD, FM and ME conducted data analysis and interpretation. KPD drafted the manuscript. FM, ME, RM, OS and KPD critically reviewed and approved the final manuscript.

\section{Funding}

Not applicable.

\begin{abstract}
Author details
${ }^{1}$ Chair of Health Management, Institute of Management (IFM), Friedrich-Alexander-Universität Erlangen-Nürnberg (FAU), Lange Gasse 20, 90403 Nuremberg, Germany. ${ }^{2}$ Institute of Experimental and Clinical Pharmacology and Toxicology, Friedrich-Alexander-Universität Erlangen-Nürnberg (FAU), Fahrstr. 17, 91054 Erlangen, Germany. ${ }^{3}$ Junior Professor for Health Services Management, Institute of Management (IFM), Friedrich-Alexander-Universität Erlangen-Nürnberg (FAU), Lange Gasse 20, 90403 Nuremberg, Germany.
\end{abstract}

Received: 10 June 2015 Accepted: 29 March 2016

Published online: 01 April 2016

\section{References}

1. Cahir C, Bennett K, Tejjeu C, Fahey T. Potentially inappropriate prescribing and adverse health outcomes in community dwelling older patients. $\mathrm{Br} J$ Clin Pharmacol. 2014;77:201-10.

2. Dalleur O, Spinewine A, Henrard S, Losseau C, Speybroeck N, Boland B. Inappropriate prescribing and related hospital admissions in frail older persons according to the STOPP and START criteria. Drugs Aging. 2012;29: 829-37.

3. Chen Y, Hwang S, Lai H, Chen T, Lin M, Chen L, et al. Potentially inappropriate medication for emergency department visits by elderly patients in Taiwan. Pharmacoepidemiol Drug Saf. 2009;18:53-61.

4. Beers MH, Ouslander JG, Rollingher I, Reuben DB, Brooks J, Beck JC. Explicit criteria for determining inappropriate medication use in nursing home residents. UCLA division of geriatric medicine. Arch Intern Med. 1991;151: 1825-32.

5. McLeod PJ, Huang AR, Tamblyn RM, Gayton DC. Defining inappropriate practices in prescribing for elderly people: a national consensus panel. CMAJ. 1997;156:385-91.

6. Laroche M, Charmes J, Merle L. Potentially inappropriate medications in the elderly: a French consensus panel list. Eur J Clin Pharmacol. 2007;63:725-31.

7. Ryan C, O'Mahony D, Kennedy J, Weedle P, Barry P, Gallagher P, et al. Appropriate prescribing in the elderly: an investigation of two screening tools, Beers criteria considering diagnosis and independent of diagnosis and improved prescribing in the elderly tool to identify inappropriate use of medicines in the elderly in primary care in Ireland. J Clin Pharm Ther. 2009; 34:369-76.

8. Akazawa M, Imai H, Igarashi A, Tsutani K. Potentially inappropriate medication use in elderly Japanese patients. Am J Geriatr Pharmacother. 2010:8:146-60.

9. Holt S, Schmiedl S, Thurmann PA. Potentially inappropriate medications in the elderly: the PRISCUS list. Dtsch Arztebl Int. 2010;107:543-51.

10. Holt S, Schmiedl S, Thurmann PA. PRISCUS-Liste potenziell inadäquater Medikation für ältere Menschen. 2011. http://priscus.net/download/PRISCUSListe_PRISCUS-TP3_2011.pdf. Accessed 31 Mar 2016.

11. Thiem U, Hinrichs T, Muller CA, Holt-Noreiks S, Nagl A, Bucchi C, et al. Prerequisites for a new health care model for elderly people with multiple morbidities: results and conclusions from 3 years of research in the PRISCUS consortium. Z Gerontol Geriatr. 2011;44 Suppl 2:101-12.

12. Siebert S, Elkeles B, Hempel G, Kruse J, Smollich M. The PRISCUS list in clinical routine: Practicability and comparison to international PIM lists. Z Gerontol Geriatr. 2012:46:35-47.

13. Schubert I, Kupper-Nybelen J, Ihle P, Thurmann P. Prescribing potentially inappropriate medication (PIM) in Germany's elderly as indicated by the PRISCUS list. An analysis based on regional claims data. Pharmacoepidemiol Drug Saf. 2013;22:719-27.

14. Dormann $\mathrm{H}$, Sonst A, Muller F, Vogler R, Patapovas A, Pfistermeister B, et al. Adverse drug events in older patients admitted as an emergency: the role of potentially inappropriate medication in elderly people (PRISCUS). Dtsch Arztebl Int. 2013;110:213-9.

15. Wissenschaftliches Institut der AOK. WIdO - Das Wissenschaftliche Institut der AOK. 2016. http://www.wido.de/wido_im_ueberblick.html. Accessed 31 Mar 2016.

16. Schwabe U, Paffrath D. Arzneiverordnungs-Report 2010: Aktuelle Daten, Kosten, Trends und Kommentare. 1st ed. Berlin: Springer; 2010.

17. Amann U, Schmedt N, Garbe E. Prescribing of potentially inappropriate medications for the elderly: an analysis based on the PRISCUS list. Dtsch Arztebl Int. 2012;109:69-75.

18. Lochner S, Kirch W, Schindler C. Managing hypertension among nursinghome residents and community-dwelling elderly in Germany: a comparative pharmacoepidemiological study. Eur J Clin Pharmacol. 2012;68:867-75.

19. Thiem U, Theile G, Junius-Walker U, Holt S, Thurmann P, Hinrichs T, et al. Prerequisites for a new health care model for elderly people with multimorbidity: the PRISCUS research consortium. Z Gerontol Geriatr. 2011;44:115-20.

20. Fiss T, Thyrian JR, Fendrich $\mathrm{K}$, van den Berg N, Hoffmann W. Cognitive impairment in primary ambulatory health care: pharmacotherapy and the use of potentially inappropriate medicine. Int J Geriatr Psychiatry. 2013;28:173-81.

21. Zimmermann $T$, Kaduszkiewicz $H$, van den Bussche $H$, Schon $G$, Brettschneider $\mathrm{C}$. Konig $\mathrm{H}$, et al. Potentially inappropriate medication in elderly primary care patients: a retrospective, longitudinal analysis. Bundesgesundheitsblatt Gesundheitsforschung Gesundheitsschutz. 2013;56:941-9.

22. Fick DM, Mion LC, Beers MH, Waller LJ. Health outcomes associated with potentially inappropriate medication use in older adults. Res Nurs Health. 2008:31:42-51

23. Stockl KM, Le L, Zhang S, Harada AS. Clinical and economic outcomes associated with potentially inappropriate prescribing in the elderly. Am J Manag Care. 2010;16:e1-10.

24. Fu AZ, Jiang JZ, Reeves JH, Fincham JE, Liu GG, Perri 3rd M. Potentially inappropriate medication use and healthcare expenditures in the US community-dwelling elderly. Med Care. 2007:45:472-6.

25. Fick DM, Waller JL, Maclean JR, Heuvel RV, Tadlock JG, Gottlieb M, et al. Potentially inappropriate medication use in a medicare managed care population: association with higher costs and utilization. J Manag Care Pharm. 2001;7:407-13.

26. United States General Accounting Office. Prescription Drugs And The Elderly: Many still receive potentially harmful drugs despite recent improvements. Washington: United States General Accounting Office; 1995. 
27. Bradley MC, Fahey T, Cahir C, Bennett K, O'Reilly D, Parsons C, et al. Potentially inappropriate prescribing and cost outcomes for older people: a cross-sectional study using the Northern Ireland enhanced prescribing database. Eur J Clin Pharmacol. 2012:68:1425-33.

28. Cahir C, Fahey T, Teeling M, Teljeur C, Feely J, Bennett K. Potentially inappropriate prescribing and cost outcomes for older people: a national population study. Br J Clin Pharmacol. 2010;69:543-52.

29. Leikola S, Dimitrow M, Lyles A, Pitkala K, Airaksinen M. Potentially inappropriate medication use among Finnish non-institutionalized people aged < $=65$ years: a register-based, cross-sectional, national study. Drugs Aging. 2011;28:227-36.

30. Al-Omar HA, Al-Sultan MS, Abu-Auda HS. Prescribing of potentially inappropriate medications among the elderly population in an ambulatory care setting in a Saudi military hospital: Trend and cost. Geriatr Gerontol Int. 2013:13:616-21.

31. Blozik E, Rapold R, von Overbeck J, Reich O. Polypharmacy and potentially inappropriate medication in the adult, community-dwelling population in Switzerland. Drugs Aging. 2013;30:561-8.

32. Gallagher P, Ryan C, Byrne S, Kennedy J, O'mahony D. STOPP (Screening Tool of Older Person's Prescriptions) and START (Screening Tool to Alert doctors to Right Treatment). Consensus validation. Int J Clin Pharmacol Ther. 2008:46:72-83.

33. Fick DM, Cooper JW, Wade WE, Waller JL, Maclean JR, Beers MH. Updating the Beers criteria for potentially inappropriate medication use in older adults: results of a US consensus panel of experts. Arch Intern Med. 2003; 163:2716-24.

34. Berdot S, Bertrand M, Dartigues J, Fourrier A, Tavernier B, Ritchie K, Alperovitch A. Inappropriate medication use and risk of falls-a prospective study in a large community-dwelling elderly cohort. BMC Geriatr. 2009;9:30.

35. Meier F, Maas R, Sonst A, Patapovas A, Müller F, Plank-Kiegele B, et al. Adverse drug events in patients admitted to an emergency department: an analysis of direct costs. Pharmacoepidemiol Drug Saf. 2015;24:176-86.

36. Albert SM, Colombi A, Hanlon J. Potentially inappropriate medications and risk of hospitalization in retirees: analysis of a US retiree health claims database. Drugs Aging. 2010;27:407-15.

37. Fillenbaum GG, Hanlon JT, Landerman LR, Artz MB, O'Connor H, Dowd B, et al. Impact of inappropriate drug use on health services utilization among representative older community-dwelling residents. Am J Geriatr Pharmacother. 2004;2:92-101.

38. Klarin I, Wimo A, Fastbom J. The association of inappropriate drug use with hospitalisation and mortality: a population-based study of the very old. Drugs Aging. 2005;22:69-82.

39. Price SD, Holman C, D'Arcy J, Sanfilippo FM, Emery JD. Association between potentially inappropriate medications from the Beers criteria and the risk of unplanned hospitalization in elderly patients. Ann Pharmacother. 2014;48:6-16.

40. van der Stelt CAK, Windsant-van den Tweel Vermeulen AMA, Egberts ACG, van den Bemt PMLA, Leendertse AJ, Hermens WAJJ, van Marum RJ, Derijks $\mathrm{HJ}$. The association between potentially inappropriate prescribing and medication-related hospital admissions in older patients: a nested case control study. Drug Saf. 2016:39:79-87.

41. Henschel F, Redaelli M, Siegel M, Stock S. Correlation of incident potentially inappropriate medication prescriptions and hospitalization: an analysis based on the PRISCUS list. Drugs - Real World Outcomes. 2015;2:249-59.

\section{Submit your next manuscript to BioMed Central and we will help you at every step:}

- We accept pre-submission inquiries

- Our selector tool helps you to find the most relevant journal

- We provide round the clock customer support

- Convenient online submission

- Thorough peer review

- Inclusion in PubMed and all major indexing services

- Maximum visibility for your research

Submit your manuscript at www.biomedcentral.com/submit
Biomed Central 